\title{
Résumé de la résistance aux antituberculeux au Canada, 2003 à 2013
}

\author{
McGuire $M^{1}$, Gallant $V^{1}$, Bourgeois A-C ${ }^{1}$, Ogunnaike-Cooke $S^{1 *}$ \\ ${ }^{1}$ Section de la surveillance du VIH/sida et de la tuberculose, Agence de la santé publique du Canada, Ottawa (Ontario) \\ * Correspondance : Susanna.Ogunnaike-Cooke@phac-aspc.gc.ca
}

\section{Résumé}

Contexte : Les souches de bacilles tuberculeux pharmacorésistantes menacent grandement de saper les efforts de prévention et de lutte contre la tuberculose. En réponse à cette inquiétude grandissante à l'échelle mondiale, l'Agence de la santé publique du Canada (l'Agence) a mis sur pied le Système canadien de surveillance des laboratoires de tuberculose (SCSLT) en association avec le Réseau technique canadien des laboratoires de tuberculose (RTCLT) et les laboratoires participants.

Objectif : Présenter les tendances et les profils de résistance aux antituberculeux au Canada de 2003 à 2013.

Méthodologie : Au début de chaque année civile, les laboratoires participants transmettent à l'Agence les résultats des épreuves de sensibilité aux antituberculeux effectués pour tous les isolats au cours de l'année précédente. Ces données sont ensuite analysées par l'Agence, et les résultats sont validés par les laboratoires participants. Enfin, les résultats sont publiés annuellement dans la série La tuberculose : La résistance aux antituberculeux au Canada.

Résultats : En 2013, les résultats des épreuves de sensibilité aux antituberculeux de 1380 isolats ont été communiqués à l'Agence. De ce nombre, 762 (54\%) étaient des isolats du complexe Mycobacterium tuberculosis, dont l'espèce était inconnue. Les deux tiers (68 \%) de l'ensemble des isolats déclarés provenaient des trois plus grandes provinces du pays, à savoir la Colombie-Britannique, I'Ontario et le Québec. Parmi tous les isolats testés par les laboratoires, $112(8,1 \%)$ présentaient une résistance à au moins un médicament majeur, et la majorité de ceux-ci (93 ou $83 \%$ ) étaient monorésistants.

Conclusion : La résistance aux antituberculeux au Canada demeure bien en deçà de la moyenne mondiale. Au cours des 10 dernières années, le pourcentage d'isolats présentant une résistance à un ou plusieurs médicaments majeurs a connu une baisse, soit de 10,5 \% en 2003 à 8,1 \% en 2013.

\section{Introduction}

Les souches de bacilles tuberculeux pharmacorésistantes menacent grandement de saper les efforts de prévention et de lutte contre la tuberculose. Bien que la tuberculose pharmacorésistante ne soit pas encore considérée comme un problème majeur au Canada, il est très possible qu'elle le devienne en raison de la fréquence à laquelle les Canadiens voyagent à l'étranger.

En 1998, en réponse à une inquiétude grandissante à l'échelle mondiale à l'égard de la résistance aux antituberculeux, le gouvernement du Canada, en collaboration avec le Réseau technique canadien des laboratoires de tuberculose (un réseau de laboratoires de tuberculose provinciaux et territoriaux composé de représentants des directeurs techniques ou scientifiques des programmes de lutte contre la tuberculose), a mis sur pied le Système canadien de surveillance des laboratoires de tuberculose (SCSLT) afin de surveiller les tendances et les profils de résistance aux antituberculeux au fil du temps. Le présent article fournit un aperçu des 
plus récentes données sur la résistance aux antituberculeux au Canada ainsi que des renseignements sur le niveau global de résistance aux antituberculeux au pays pour la période de 2003 à 2013, en particulier pour l'année 2013 (1).

\section{Contexte}

Le SCSLT est un système de surveillance basé sur les isolats qui vise à recueillir des données opportunes sur la résistance aux antituberculeux dans tout le Canada. Les renseignements sur les résultats de tous les isolats uniques dont la pharmacorésistance a été évaluée au cours de l'année civile précédente sont soumis volontairement par les laboratoires de tuberculose provinciaux à l'Agence aux fins d'intégration dans le Système. Les objectifs principaux de ce système sont les suivants :

- Déterminer annuellement le nombre d'isolats de bacilles tuberculeux pharmacorésistants au Canada selon le profil de résistance, la province ou le territoire, le sexe et l'âge.

- Surveiller les tendances en matière de tuberculose pharmacorésistante au Canada au fil du temps selon la province ou le territoire, le sexe et l'âge.

La résistance aux antituberculeux est déterminée au moyen d'épreuves de sensibilité des cultures d'échantillons cliniques prélevés chez des personnes atteintes de tuberculose à culture positive (2). On dit que les personnes atteintes de tuberculose active présentent une tuberculose pharmacorésistante si la souche du complexe Mycobacterium tuberculosis responsable de leur maladie est résistante à un ou plusieurs des quatre antituberculeux majeurs : isoniazide, rifampicine, éthambutol ou pyrazinamide. Les données sont soumises pour les cas confirmés pour lesquels le complexe Mycobacterium tuberculosis, notamment Mycobacterium tuberculosis, M. africanum, M. canetti, M. caprae, M. microti, M. pinnipedii ou M. bovis, à l'exception du bacille de Calmette-Guérin (BCG) de $M$. bovis, a été mis en évidence. Consulter les définitions des différents profils de pharmacorésistance dans l'encadré ci-dessous.

\section{Définitions des différents profils de tuberculose pharmacorésistante}

\section{Monorésistance}

- Résistance à un seul antituberculeux majeur

\section{Polyrésistance (autres profils)}

- Résistance à plus d'un antituberculeux majeur, à l'exclusion de la résistance à la fois à l'isoniazide et à la rifampicine qui correspond à la tuberculose multirésistante

\section{Tuberculose multirésistante}

- Tuberculose causée par des bactéries résistantes à l'isoniazide et à la rifampicine avec ou sans résistance à d'autres antituberculeux

\section{Tuberculose ultrarésistante}

- Tuberculose causée par des bactéries résistantes à l'isoniazide et à la rifampicine ainsi qu'à toute fluoroquinolone et à au moins un des trois antituberculeux injectables mineurs (amikacine, capréomycine ou kanamycine)

\section{Méthodologie}

Au début de chaque année civile, les laboratoires participants soumettent volontairement les résultats des épreuves de sensibilité effectués pour tous les isolats du complexe Mycobacterium tuberculosis au cours de 
l'année précédente. Même si certains laboratoires soumettent les résultats pour la souche de Calmette-Guérin (BCG) de M. bovis, ils ne font pas partie du présent article.

Les renseignements suivants sont transmis à l'Agence : la date à laquelle l'isolat ou le spécimen a été reçu au laboratoire et son numéro d'identification; la province ou le territoire où l'isolat a été testé ainsi que la province ou le territoire originaire; le sexe et la date de naissance (ou l'âge) de la personne de qui provient l'isolat; et les résultats des épreuves de sensibilité.

En ce qui concerne les résultats des épreuves de sensibilité, les laboratoires doivent soumettre les résultats pour tous les antituberculeux majeurs (isoniazide [INH], rifampicine [RMP], éthambutol [EMB] et pyrazinamide [PZA]). Si les isolats présentent une résistance à l'isoniazide et à la rifampicine, une épreuve de sensibilité aux antituberculeux mineurs devrait être effectuée pour éliminer la tuberculose ultrarésistante (2). Les laboratoires doivent soumettre les résultats des épreuves de sensibilité pour une fluoroquinolone (ofloxacine, moxifloxacine ou lévofloxacine) et pour les médicaments injectables (amikacine, kanamycine et capréomycine).

L'analyse présentée ici est uniquement fondée sur des statistiques descriptives. Aucune procédure statistique n'a été utilisée pour les analyses comparatives, et aucune technique statistique n'a été appliquée pour tenir compte des données manquantes. Les données des tableaux contenant de petites cellules $(n=\leq 5)$ n'ont pas été supprimées puisqu'elles ne semblaient pas présenter de risque d'identification de cas individuels. Ces procédures sont conformes à la directive de l'Agence sur la collecte, l'utilisation et la diffusion de l'information sur la santé publique (3).

\section{Résultats}

\section{Résultats de 2013}

En 2013, les résultats des épreuves de sensibilité aux antituberculeux ont été présentés à l'Agence pour 1397 isolats. Parmi ces isolats, 762 (54 \%) faisaient partie du complexe Mycobacterium tuberculosis, dont l'espèce était connue (750 ont été identifiés comme $M$. tuberculosis, 8 comme $M$. africanum et 4 comme M. bovis), et 618 (44 \%) faisaient partie du complexe Mycobacterium tuberculosis, mais dont l'espèce était inconnue. Dix-sept isolats $(1,2 \%)$ étaient identifiés comme $M$. bovis BCG et ont été exclus des analyses ultérieures (données non représentées). Par conséquent, les résultats de laboratoire de 1380 isolats ont été analysés aux fins du présent article. De ces isolats déclarés, $68 \%$ provenaient des trois plus grandes provinces du pays, à savoir la Colombie-Britannique, l'Ontario et le Québec. La Figure 1 présente la répartition du nombre d'isolats testés par province ou territoire d'origine. 
Figure 1 : Nombre d'isolats du complexe Mycobacterium tuberculosis testés par province ou territoire d'origine, Canada, 2013

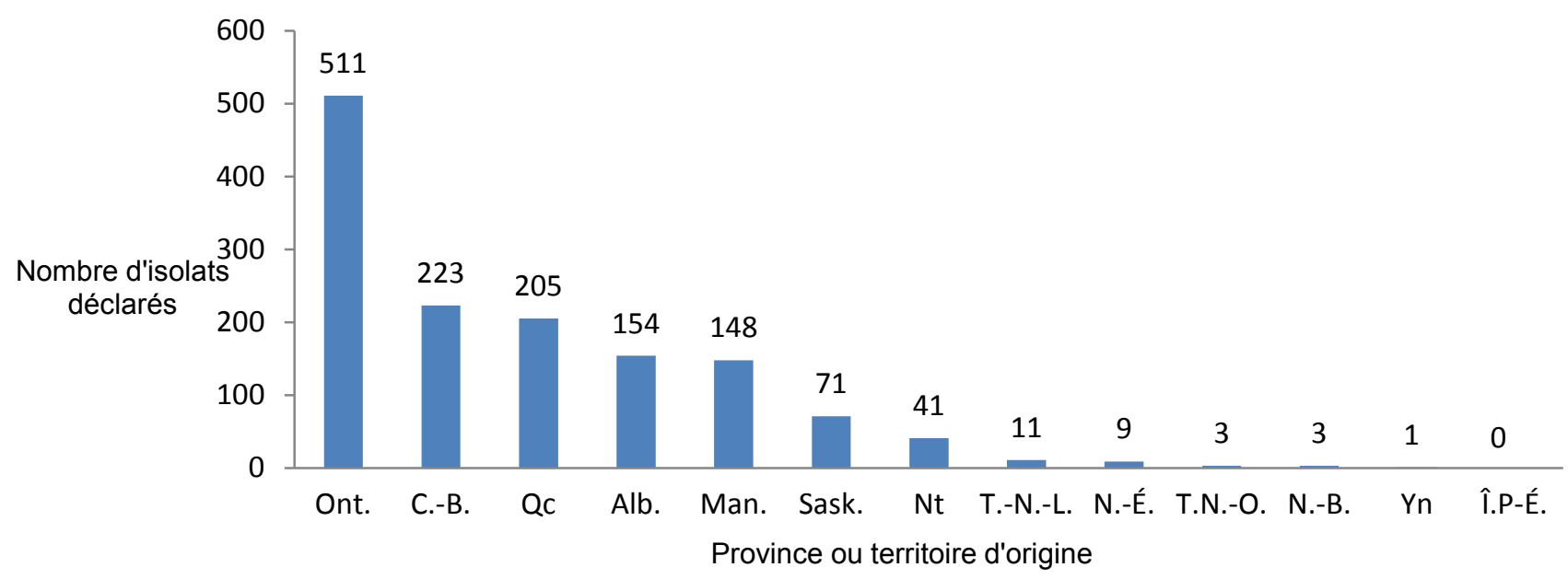

\section{Monorésistance}

En 2013, sur les 112 isolats de bacilles tuberculeux déclarés résistants à au moins un des quatre antituberculeux majeurs, la majorité, soit 93 isolats (83\%), étaient monorésistants (Tableau 3). De ce nombre, 74 (80\%) étaient résistants à l'isoniazide, $17(18 \%)$ étaient résistants au pyrazinamide et $2(2 \%)$ étaient résistants à la rifampicine. Aucun isolat n'a été identifié comme étant résistant à l'éthambutol (données non représentées). La Figure 2 présente la répartition du pourcentage de tous les isolats testés qui étaient résistants à l'un ou l'autre des quatre antituberculeux majeurs.

Figure 2 : Résistance aux antituberculeux majeurs déclarée au Canada par type de médicament, 2013

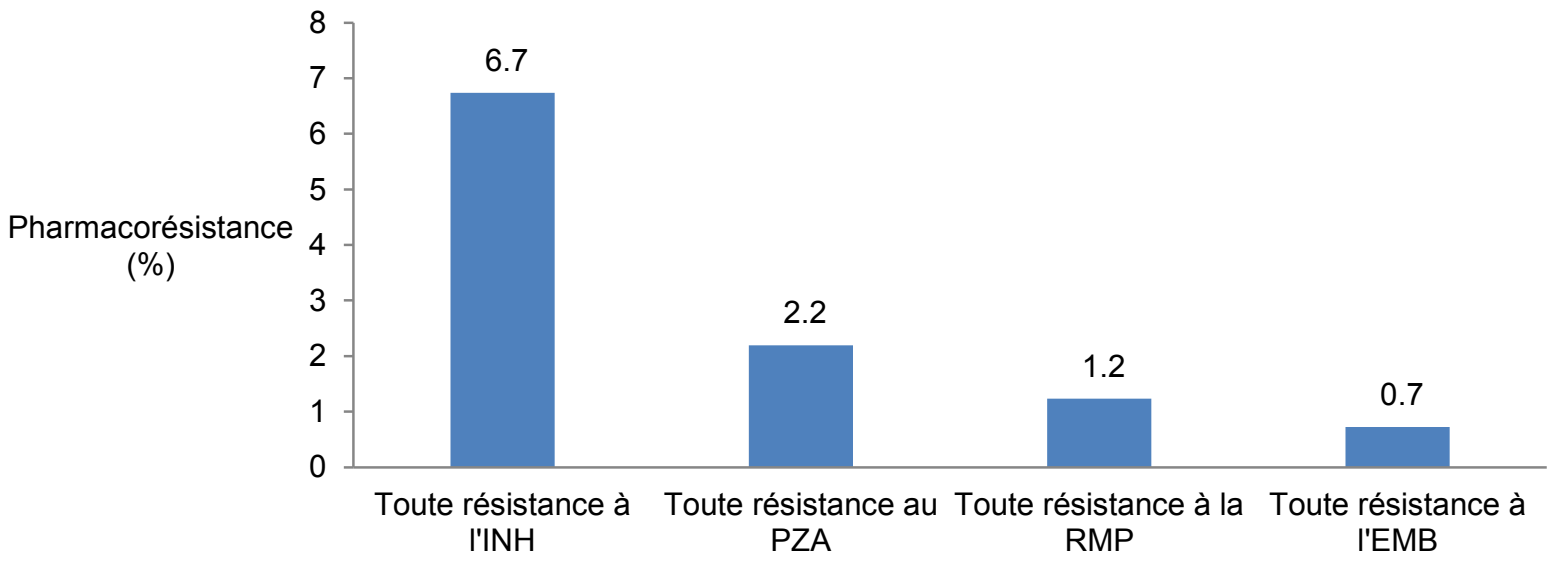

Type de pharmacorésistance

\section{Multirésistance}

En 2013, 15 isolats étaient résistants à la fois à l'isoniazide et à la rifampicine (ce qui permet de les classer comme au moins multirésistants), et six (40\%) d'entre eux étaient résistants uniquement à l'isoniazide et à la rifampicine, mais à aucun autre antituberculeux majeur. En plus d'être résistants à l'isoniazide et à la rifampicine, trois $(20 \%)$ de ces 15 isolats étaient également résistants à l'éthambutol, un (6\%) au pyrazinamide et cinq 
(33 \%) à tous les antituberculeux majeurs. Quatre autres isolats $(0,3 \%)$ étaient résistants à au moins deux antituberculeux majeurs, à l'exclusion de la résistance à la fois à l'isoniazide et à la rifampicine.

\section{Ultrarésistance}

Afin d'écarter la possibilité de tuberculose ultrarésistante, les 15 isolats qui étaient résistants à la fois à l'isoniazide et à la rifampicine ont tous été soumis ensuite à des épreuves de sensibilité à certains antituberculeux mineurs. De ce nombre, 12 isolats étaient sensibles à la fois aux antituberculeux injectables et aux fluoroquinolones, un isolat était résistant à un antituberculeux injectable et un isolat était résistant à une fluoroquinolone. Par conséquent, ces 14 isolats $(1,0 \%$ de l'ensemble des isolats testés) ont été classés comme multirésistants. L'isolat restant était résistant à au moins un des antituberculeux injectables et à une fluoroquinolone, ce qui en faisait un isolat ultrarésistant $(0,1 \%$ de l'ensemble des isolats testés). Ainsi, les résultats des épreuves supplémentaires de sensibilité aux antituberculeux mineurs en 2013 ont permis d'identifier 14 isolats de tuberculose multirésistante et un isolat de tuberculose ultrarésistante (Figure 3).

Figure 3 : Profils de résistance aux antituberculeux déclarée sous forme de pourcentage des isolats testés, 2013

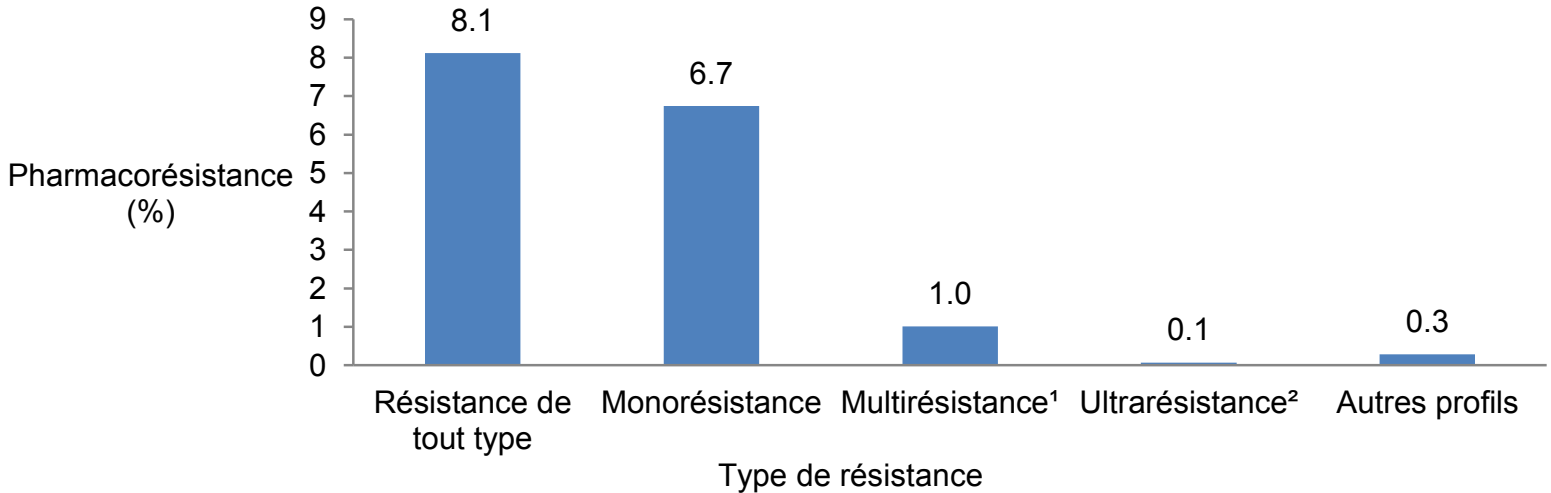

\footnotetext{
${ }^{1}$ La tuberculose multirésistante est une tuberculose qui est résistante, à tout le moins, à l'isoniazide et à la rifampicine, mais qui ne répond pas à la définition de tuberculose ultrarésistante.

${ }^{2}$ La tuberculose ultrarésistante (TB-UR) est une tuberculose qui est résistante, à tout le moins, aux deux meilleurs antituberculeux majeurs, soit l'isoniazide et la rifampicine, en plus d'être résistante à des antituberculeux mineurs, y compris à toute fluoroquinolone et à au moins un des trois antituberculeux mineurs injectables (amikacine, capréomycine et kanamycine).
}

\section{Distribution géographique}

En 2013, 68 \% de tous les isolats déclarés provenaient des trois plus grandes provinces du pays, à savoir la Colombie-Britannique, l'Ontario et le Québec. Tous les isolats provenant des Territoires du Nord-Ouest, du Nunavut, du Yukon et de Terre-Neuve-et-Labrador étaient complètement sensibles à tous les antituberculeux majeurs testés. L'Île-du-Prince-Édouard n'a signalé aucun cas de tuberculose en 2013, et aucun résultat de laboratoire n'a été déclaré par cette province. Sur les 15 isolats résistants à la fois à l'isoniazide et à la rifampicine en 2013, 13 provenaient de l'Ontario et deux du Québec. 


\section{Données démographiques}

En 2013, des renseignements sur le sexe ont été déclarés pour toutes les personnes de qui provenaient les isolats, sauf deux personnes, et l'âge ou la date de naissance était indiqué dans tous les cas. Les hommes représentaient $55 \%$ de tous les cas rapportés. La Figure 4 montre le nombre d'isolats testés selon le groupe d'âge et le sexe.

Figure 4 : Tous les isolats testés selon le sexe et le groupe d'âge en 2013

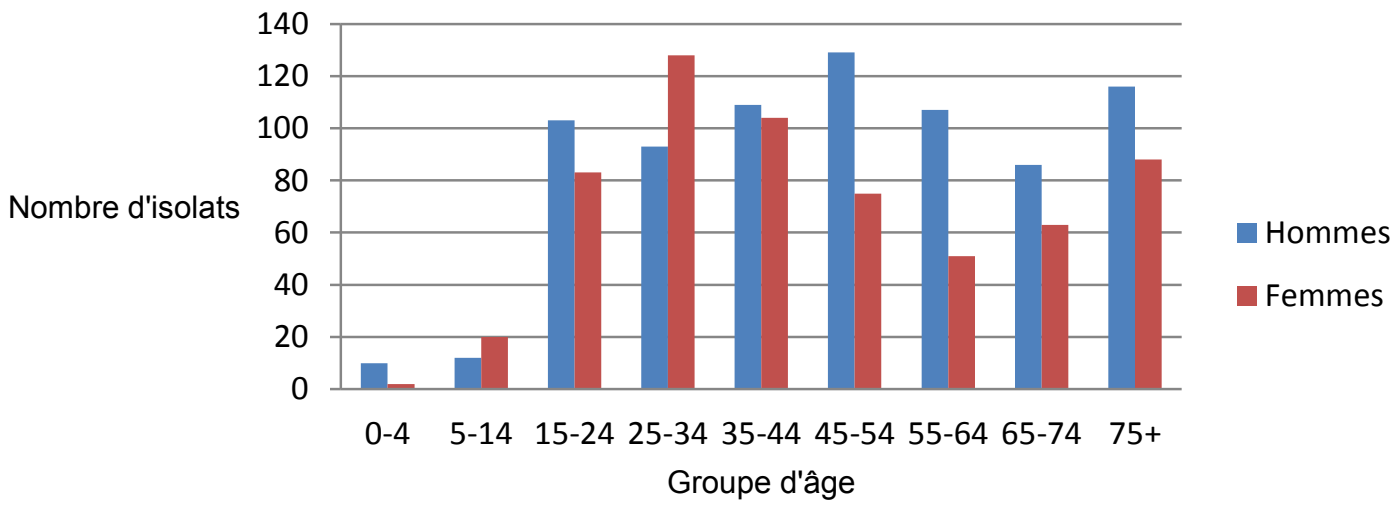

Sur les 112 isolats pour lesquels un profil de résistance a été déterminé, $23 \%$ provenaient de personnes âgées de 24 à 34 ans et $21 \%$, de personnes âgées de 45 à 54 ans. Moins de $2 \%$ des isolats ont été prélevés chez des personnes âgées de moins de 15 ans. Tous les isolats de tuberculose multirésistante provenaient de personnes âgées de 15 à 64 ans. Aucun cas de tuberculose multirésistante n'a été signalé chez des personnes âgées de moins de 15 ans ou de plus de 65 ans. Les femmes représentaient $56 \%$ des isolats présentant tout type de résistance et $67 \%$ (10 sur 15) des isolats résistants à la fois à l'isoniazide et à la rifampicine. La Figure 5 montre la répartition des isolats présentant tout type de résistance et les isolats de tuberculose multirésistante (TB-MR) selon le sexe et l'âge en 2013.

Figure 5 : Nombre d'isolats présentant une résistance selon le groupe d'âge et le sexe en 2013

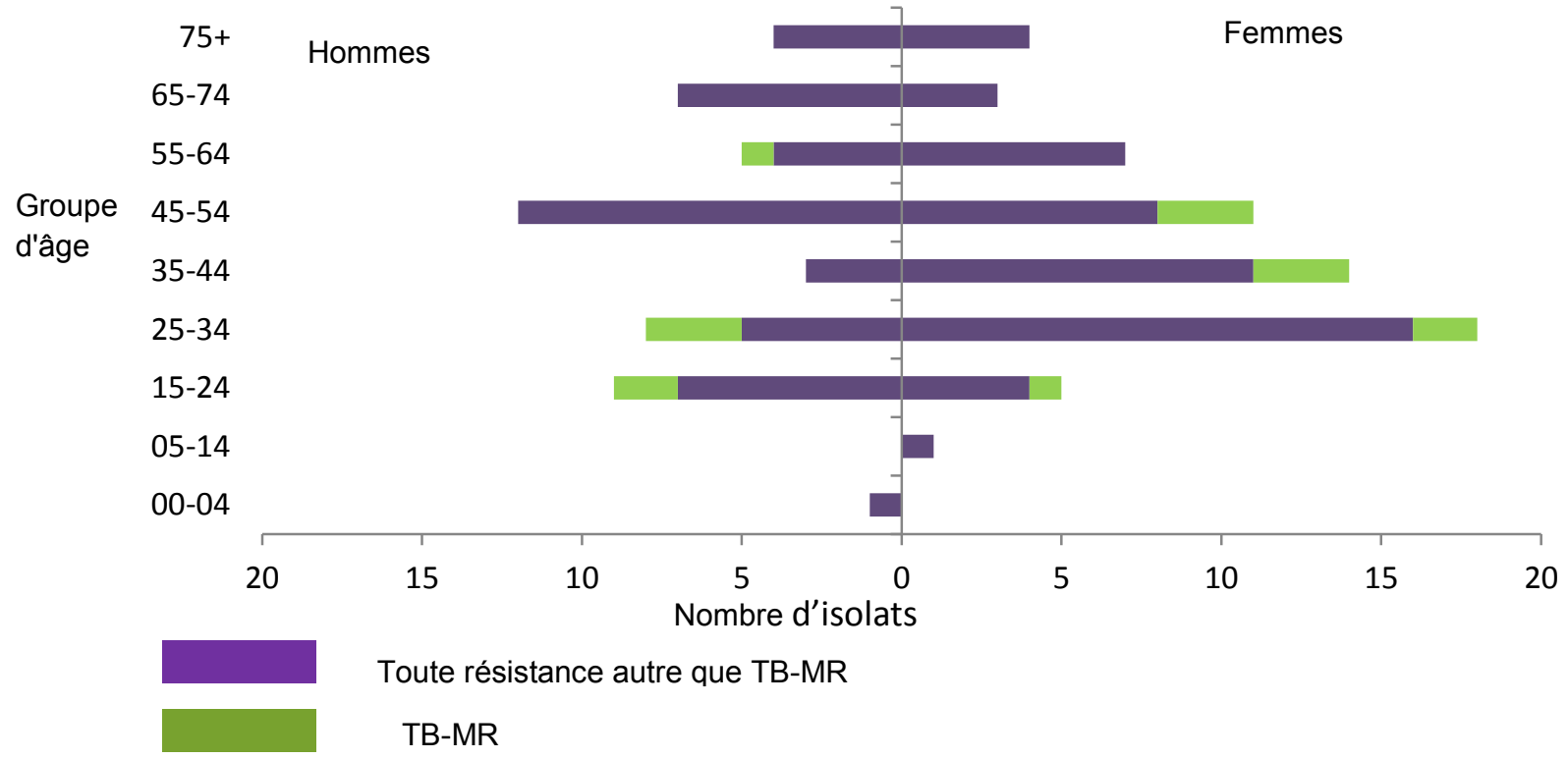


Tendances : 2003 à 2013

Pour la période de 2003 à 2013, des résultats d'épreuves de sensibilité ont été déclarés pour 14841 isolats. En moyenne, 1349 résultats ont été reçus chaque année, avec un maximum de 1405 en 2003 et un minimum de 1267 en 2007.

Au cours de la même période, 8,0 \% (plage comprise entre 6,7 \% et 9,4\%) de l'ensemble des isolats testés étaient résistants à l'isoniazide. Entre 2011 et 2013, la résistance à l'isoniazide a légèrement diminué, passant de $9,2 \%$ en 2011 à $7,9 \%$ en 2012, puis à son niveau historique le plus bas avec $6,7 \%$ en 2013. Pour la période de 2003 à 2013, la résistance à l'éthambutol, à la rifampicine et au pyrazinamide est systématiquement restée inférieure à 3,0 \% (Figure 6).

Figure 6 : Tout type de pharmacorésistance par type d'antituberculeux majeur, sous forme de pourcentage des isolats testés, 2003 à 2013

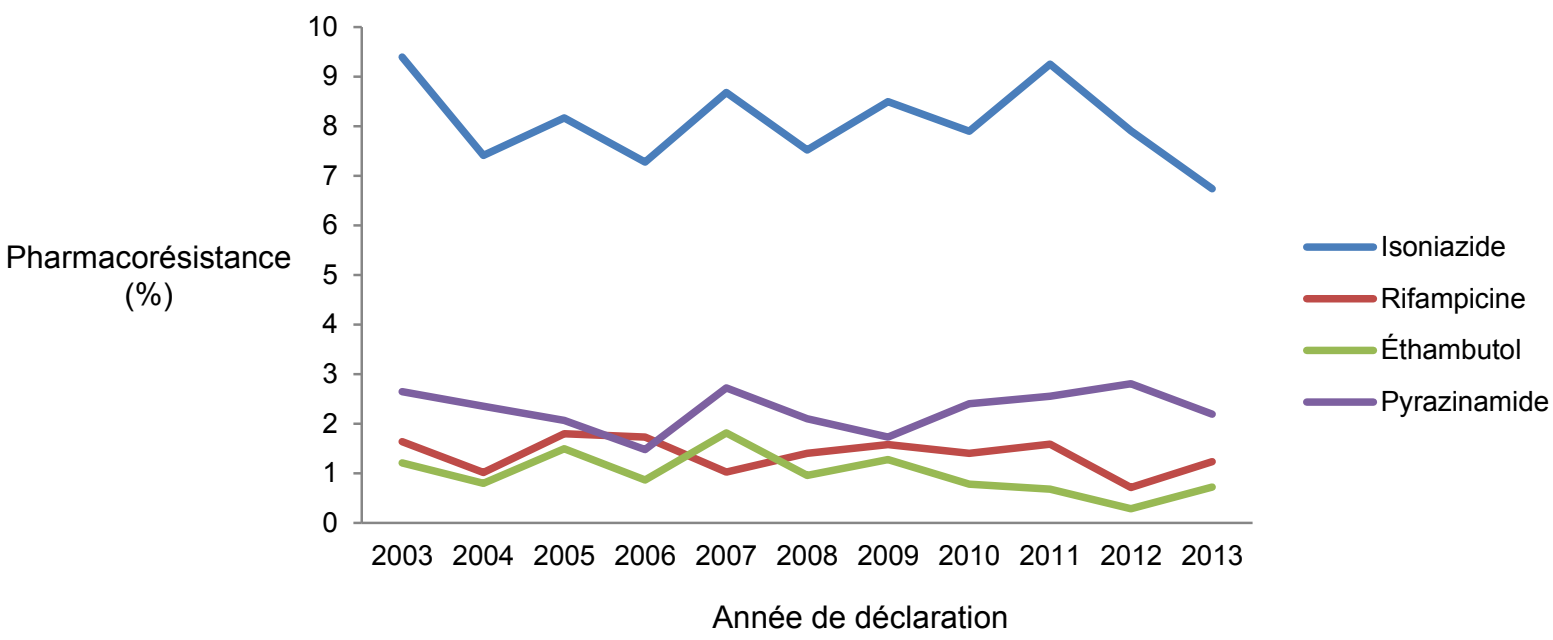

Parmi tous les résultats déclarés entre 2003 et 2013, 1399 isolats (environ $9 \%$ ) étaient résistants à un ou plusieurs antituberculeux majeurs, le niveau le plus haut, à 10,5\%, ayant été observé en 2003, 2007 et 2011, et le niveau le plus bas, à 8,1\%, en 2013.

Pour la période de 2003 à 2013, 170 isolats ont été classés comme multirésistants, ce qui représentait 1,1\% des isolats testés au cours de cette période. Sept isolats ont été classés comme ultrarésistants, ce qui équivaut à une moyenne annuelle inférieure à $0,1 \%$ du nombre total d'isolats testés pour la période de 2003 à 2013. En moyenne, 16 isolats de tuberculose multirésistante ont été déclarés chaque année, avec un maximum de 22 cas ( $1,6 \%$ de tous les isolats testés) en 2005 et un minimum de 8 cas (0,6\% de tous les isolats) en 2012. Pour la période de 2003 à 2013, les 170 isolats multirésistants provenaient tous de six provinces : l'Alberta, la ColombieBritannique, le Manitoba, I'Ontario, le Québec et la Saskatchewan. Sur les sept autres isolats identifiés comme ultrarésistants, cinq ont été déclarés par l'Ontario, un par le Manitoba et un par le Québec.

La Figure 7 montre le profil général de résistance aux antituberculeux déclarée sous forme de pourcentage des isolats testés pour la période de 2003 à 2013. Malgré de petites fluctuations du pourcentage d'isolats présentant divers profils de résistance, aucune évolution notable n'a été observée au cours de cette période. 
Figure 7 : Profils de résistance aux antituberculeux déclarée sous forme de pourcentage des isolats testés, 2003 à 2013

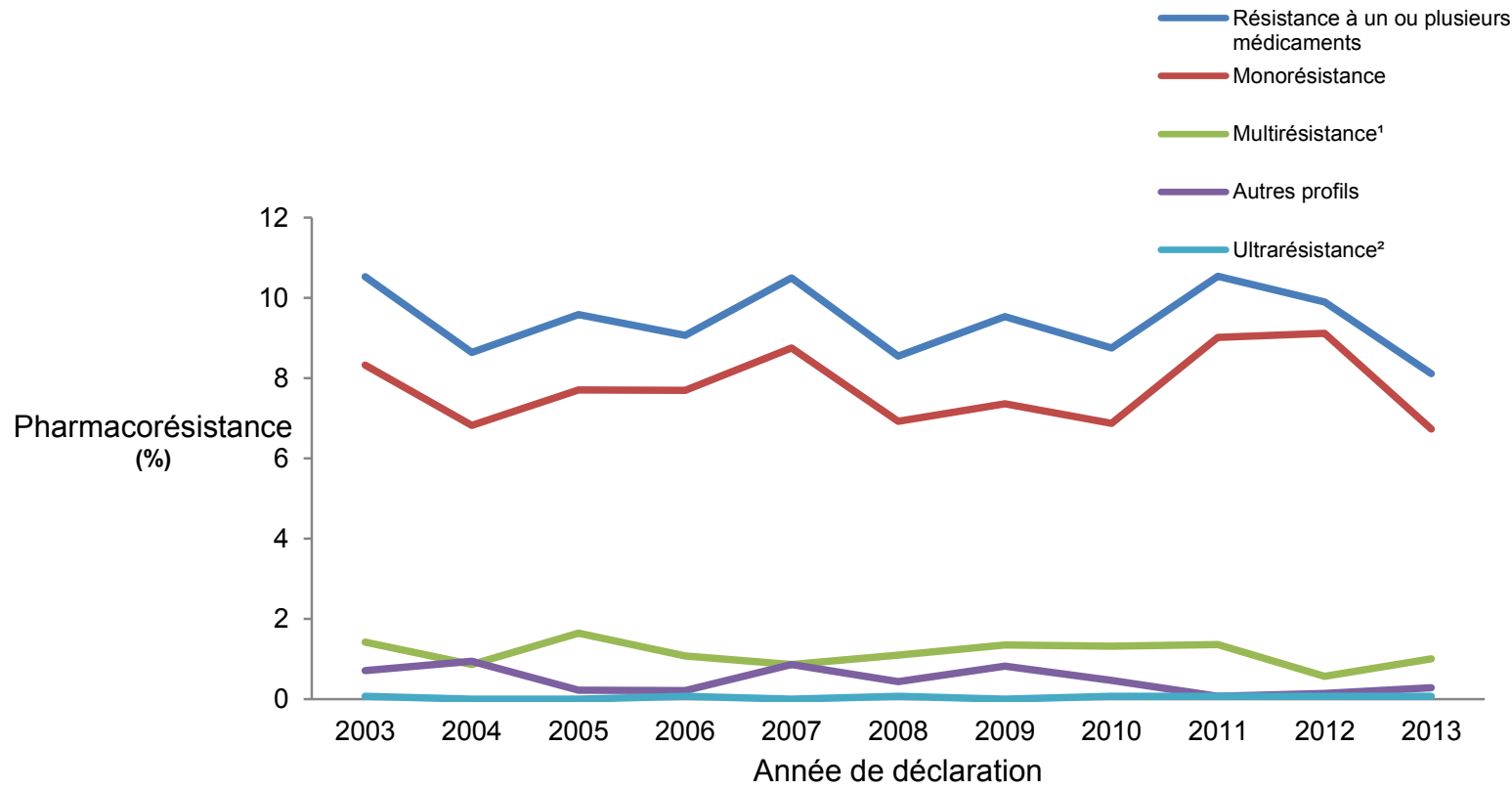

\footnotetext{
${ }^{1}$ La tuberculose multirésistante est une tuberculose qui est résistante, à tout le moins, à l'isoniazide et à la rifampicine, mais qui ne répond pas à la définition de tuberculose ultrarésistante.

${ }^{2}$ La tuberculose ultrarésistante (TB-UR) est une tuberculose qui est résistante, à tout le moins, aux deux meilleurs antituberculeux majeurs, soit l'isoniazide et la rifampicine, en plus d'être résistante à des antituberculeux mineurs, y compris à toute fluoroquinolone et à au moins un des trois antituberculeux mineurs injectables (amikacine, capréomycine et kanamycine).
}

\section{Discussion}

La résistance aux antituberculeux au Canada demeure bien en deçà de la moyenne mondiale. Au cours des 10 dernières années, le pourcentage d'isolats présentant une résistance à un ou plusieurs médicaments majeurs a connu une baisse, soit de 10,5\% en 2003 à 8,1\% en 2013.

Les organismes résistants à la fois à l'isoniazide et à la rifampicine, un profil de résistance appelé tuberculose multirésistante, rendent les efforts de traitement et de prévention considérablement plus difficiles, car les antituberculeux efficaces sont limités. Les données publiées par l'Organisation mondiale de la Santé indiquent qu'en 2012, à l'échelle mondiale, environ 3,6 \% (intervalle de confiance à $95 \%: 2,1 \%$ à 5,1 \%) des nouveaux cas de tuberculose et 20,2 \% (intervalle de confiance à $95 \%: 13,3 \%$ à 27,2 \%) des cas de tuberculose déjà traités étaient multirésistants (4). Au Canada, la proportion des cas tuberculose mutirésistante se situe à 1.1\%.

L'interprétation des résultats doit être effectuée en tenant compte de certaines limites. Compte tenu du peu de renseignements d'identification pour chaque isolat (âge et sexe de la personne), il est possible que des déclarations multiples pour une même personne soient présentes dans la base de données. Cependant, ce biais est considéré comme minime.

Habituellement, seuls les isolats de tuberculose multirésistante ou d'autres profils de tuberculose ultrarésistante font l'objet d'épreuves de sensibilité à certains antituberculeux mineurs. Bien que le Clinical and Laboratory Standards Institute recommande d'évaluer la résistance aux antituberculeux mineurs des isolats monorésistants à l'isoniazide, ainsi que des autres isolats polyrésistants mais non multirésistants, cette recommandation n'est pas 
toujours respectée au Canada. Les isolats autres que ceux de la tuberculose multirésistante peuvent être résistants à une fluoroquinolone, car cette famille d'antibiotiques est largement utilisée dans le traitement des infections respiratoires. Dans une certaine mesure, notre compréhension de l'émergence de la résistance aux antituberculeux mineurs au Canada en est donc limitée. La quantité de données démographiques et cliniques recueillies au moyen du SCSLT est minime. Par conséquent, il est impossible de distinguer une résistance primaire d'une résistance secondaire ou acquise de même que les profils de résistance divergents des nouveaux cas par rapport aux cas de retraitement. Toutefois, le rapport La tuberculose au Canada présente également des données fondées sur des cas à propos de la pharmacorésistance primaire et acquise au Canada. Ce rapport jumelé au rapport La tuberculose : La résistance aux antituberculeux au Canada fournissent un portrait exhaustif des données de surveillance des cas de tuberculose et de la résistance aux antituberculeux à l'échelle nationale.

Les données recueillies à ce jour indiquent que la présence de tuberculose pharmacorésistante au Canada est plus faible que ce qui s'observe en moyenne dans le monde et que les chiffres restent relativement stables depuis le début de la déclaration. Cependant, l'inquiétude croissante suscitée dans le monde par la résistance aux antituberculeux et par l'émergence de souches de tuberculose ultrarésistante fait ressortir le rôle vital que continue à jouer le SCSLT dans la surveillance de la résistance aux antituberculeux au Canada.

\section{Remerciements}

La Division de surveillance de la santé et de l'épidémiologie de l'Agence de la santé publique du Canada tient à remercier les membres du Réseau technique canadien de laboratoires de tuberculose et leurs équipes, ainsi que les collègues du Laboratoire national de microbiologie, pour leur contribution et leur participation au Système canadien de surveillance des laboratoires de tuberculose.

\section{Conflit d'intérêts}

Aucun

\section{Financement}

Ce travail a été appuyé par l'Agence de la santé publique du Canada.

\section{Références}

(1) Public Health Agency of Canada. Tuberculosis: Drug Resistance in Canada 2013. Ottawa, ON: Minister of Public Works and Government Services Canada; 2014. http://www.phac-aspc.gc.ca/tbpc-latb/pubs/tb-dr2013/index-eng.php.

(Disponible en français : http://www.phac-aspc.gc.ca/tbpc-latb/pubs/tb-dr2013/index-fr.php).

(2) Christianson S, Jamieson F, Sharma M, Wolfe J. Appendix D : Normes pour les laboratories de tuberculose et de mycobactériologies : services et politiques. Dans Agence de la santé publique du Canada, Société canadienne de thoracologie. Normes canadiennes pour la lutte antituberculeuse, $7^{\mathrm{e}}$ éd. Menzies R, Wong T., Ottawa, ON : Ministère de santé, 2013.

(3) Public Health Agency of Canada. Directive for the collection, use and dissemination of information relating to public health. Unpublished document. Ottawa, ON: Minister of Health, 2013

(4) World Health Organization. Global tuberculosis report 2013. Geneva: WHO Press; 2013. http://apps.who.int/iris/bitstream/10665/91355/1/9789241564656_eng.pdf 